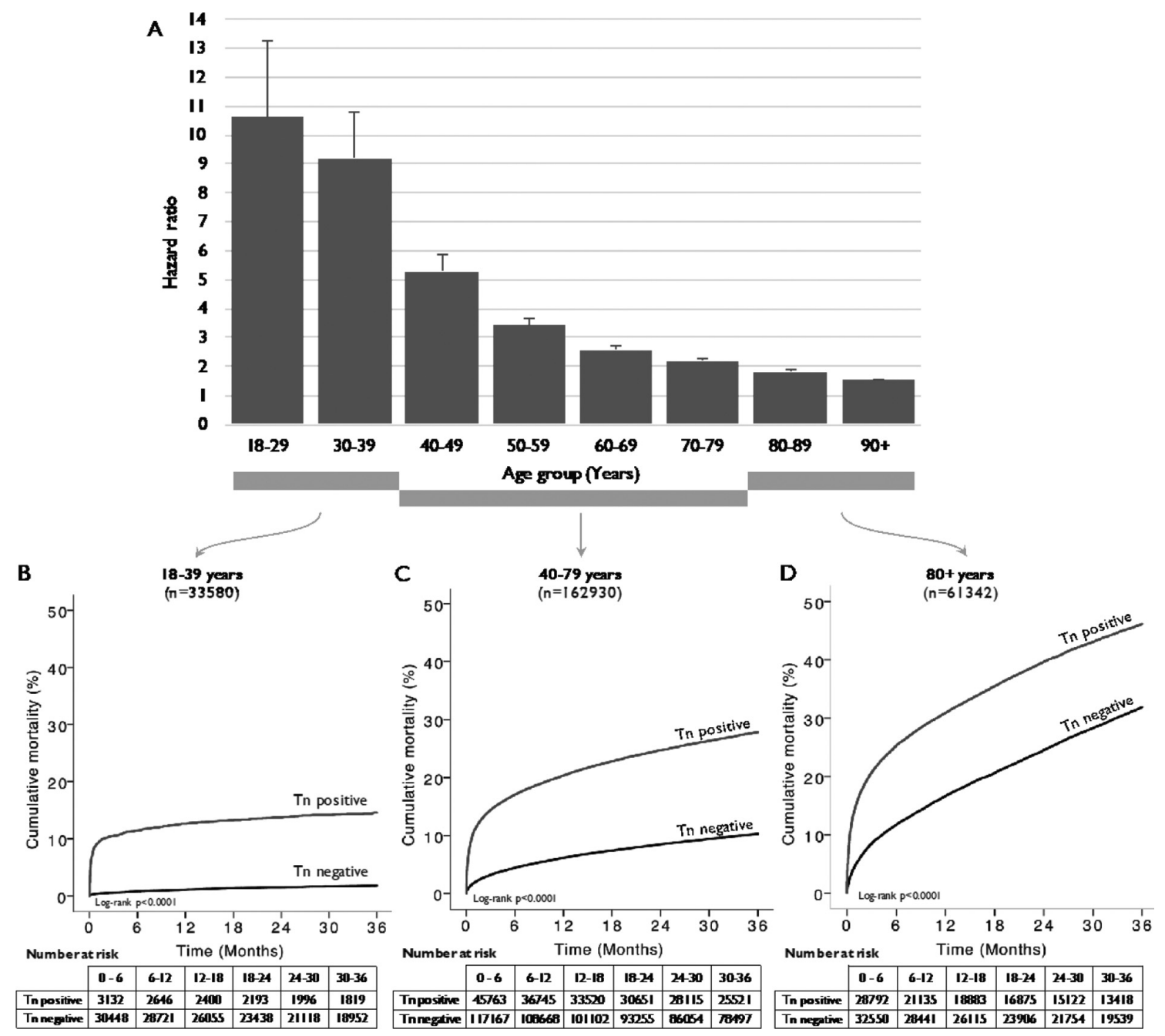

Abstract 146 Figure 1 A) Hazard ratios for troponin positive versus negative groups across different age bands for all patients; B-D, Kaplan-Meier survival curve by troponin positivity in 18-39, 40-79 and 80+ year age bands.

Error bars denote upper $95 \%$ confidence interval. Tn, troponin.

fold (95\%, CI 1.4-1.6) in the over 90s (figure 1A). Individual survival curves for troponin positive and negative are shown for the 18-39, 40-79 and 80 years age bands in figure 1B-D. Conclusion Patients in routine clinical practice who have a troponin measured have a very high mortality. While troponin positivity is predictive of mortality across the age spectrum, a troponin test being positive makes the most dramatic difference in survival in young patients. In patients over the age of 80 , a positive troponin was still an independent predictor of mortality but the incremental risk was much less than in younger age groups. A positive troponin should be taken seriously across the age spectrum, especially in young patients.

Conflict of Interest No conflict of interest

\section{ROLE OF CX3CR1HI NON-CLASSICAL MONOCYTES IN MYOCARDIAL ISCHEMIA-REPERFUSION}

${ }^{1}$ Sarah Marsh ${ }^{*},{ }^{1}$ Helen Arthur, ${ }^{2}$ loakim Spyridopoulos. ${ }^{1}$ Newcastle University; ${ }^{2}$ Institute of Genetic Medicine, Newcastle University

10.1136/heartjnl-2019-BCS.144

Introduction Aside from their patrolling behaviour, the early functions of CX3CR1hi non-classical (NC) monocytes following acute myocardial ischemia-reperfusion (I/R) are largely unknown. NC monocyte behaviour is orchestrated in part by their high expression of the fractalkine receptor, CX3CR1, however the signalling pathways activated by fractalkine stimulation remain largely undefined. Increasing evidence has now implicated the CX3CR1/CX3CL1 system in myocardial $\mathrm{I} / \mathrm{R}$ injury, which conclusively suggest that fractalkine has a detrimental effect on myocardial ischemia and accelerates the progress of heart failure. Neutralization of the receptor is successful in improving cardiac function in a mouse model of MI, and STEMI patient CX3CL1 concentration is a prognostic marker following MI. The first aim of this project was therefore to characterise circulating CX3CR1hi NC monocyte dynamics in ST elevation MI (STEMI) patients immediately following reperfusion, and evaluate their prognostic value. Using a mouse model of cardiac I/ $\mathrm{R}$, we evaluated the effect of genetic CX3CR1 knockout on non-classical monocyte recruitment into the injured myocardium at $2 \mathrm{~h}$ and $24 \mathrm{~h}$ post-I/R. Since contemporary efforts to promote myocardial repair have failed to translate into clinical therapies thus far, we hypothesize that CX3CR1 may be a prime candidate to target myocardial repair following $\mathrm{I} / \mathrm{R}$.

Method Flow Cytometry (FC) quantified monocyte subpopulations in the blood of 59 STEMI patients pre and post PPCI. 
Infarct size and microvascular obstruction (MVO) were assessed using cardiac MRI. Monocytes were quantified in a second study of 14 STEMI patients. A mouse model of cardiac $\mathrm{I} / \mathrm{R}$ injury was used to study monocyte infiltration following MI. The recruitment of classical monocytes [CD11b+ GFPmid CCR2hi] and NC monocytes [CD11b+ GFPhi CCR2lo] into the injured myocardium at $2 \mathrm{~h}$ and $24 \mathrm{~h}$ post-IR was studied by immunofluorescence in CX3CR1+/GFP CX3CR1GFP/GFP mice. FC quantified absolute monocyte subset counts in mouse blood at $2 \mathrm{~h}$ and $24 \mathrm{~h}$ post-IR.

Results STEMI patients showed a $46 \%( \pm 4)$ drop in circulating CX3CR1hi CD16 ++ NC monocytes $(\mathrm{p}<0.0001)$ at pre-90 min post-reperfusion. This was not observed for classical and intermediate monocytes ( $7 \%$ and 20\%, respectively). STEMI patients with larger infarcts and reduced LVEF post-MI showed a significantly greater decline $(p=0.039 \& p=0.043$, respectively) in CX3CR1hi $\mathrm{CD} 16++\mathrm{NC}$ monocytes. These findings were confirmed by enhanced monocyte subset quantification in a second STEMI cohort $(52 \%( \pm 6)$ NC drop, $\mathrm{p}=0.0008)$. Intra-myocardial infiltration of $\mathrm{NC}$ monocytes was determined in a CX3CR1+/GFP mouse model of cardiac I/R. At $24 \mathrm{~h}$ post-I/R, NC counts significantly increased from $2 \mathrm{~h}$ by approximately 5 -fold $(\mathrm{p}<0.0001)$. In CX3CR1GFP/GFP mice, NC monocyte infiltration was 2-fold lower compared to CX3CR1+/GFP mice, suggesting that NC monocytes may rely partly on the fractalkine receptor for myocardial infiltration post-I/R. In the circulation, there was a $70 \%$ reduction in $\mathrm{NC}$ monocytes from baseline to $2 \mathrm{~h}$ post-I/R, suggesting that $\mathrm{NC}$ mono may originate from the marginating pool of monocytes in the peripheral blood.

Conclusion \& Future Work: NC monocytes appear to specifically respond to cardiac $\mathrm{I} / \mathrm{R}$, and may be predictive of cardiac function following STEMI. The 2-fold reduction in CX3CR1hi LY6Clo NC monocyte infiltration in CX3CR1GFP/GFP mice suggests that CX3CR1 has an important function in the NC monocyte response to cardiac $\mathrm{I} / \mathrm{R}$.

Conflict of Interest non

\section{CARDIAC MYOSIN-BINDING PROTEIN C TO DIAGNOSE ACUTE MYOCARDIAL INFARCTION IN THE PRE- HOSPITAL SETTING, USING MULTI-FACTORIAL NOMOGRAMS}

${ }^{1}$ Thomas Kaier* ${ }^{2}$ Carsten Stengaard, ${ }^{1}$ lack Marjot, ${ }^{2}$ Jacob Thorsted Sørensen, ${ }^{1}$ Bashir Alaour, 'Stavroula Stavropoulou-Tatla, ${ }^{2}$ Christian Juhl Terkelsen, 'Luke Williams, ${ }^{2}$ Kristian Thygesen, ${ }^{3}$ Ekkehard Weber, ${ }^{1}$ Michael Marber, ${ }^{2}$ Hans Erik Bøtker. ${ }^{1}$ King's College London BHF Centre; ${ }^{2}$ Aarhus University Hospital; ${ }^{3}$ Martin Luther University Halle-Wittenberg

10.1136/heartjnl-2019-BCS.145

Background Early triage is essential to improve outcome in patients with suspected Acute Myocardial Infarction (AMI). This study investigated whether cardiac myosin-binding protein $\mathrm{C}$ (cMyC), a novel biomarker of myocardial necrosis, can aid early diagnosis of AMI and risk stratification.

Methods cMyC and hs-cTnT were retrospectively quantified in blood samples obtained by ambulance-based paramedics in a prospective, diagnostic cohort study. Patients with ongoing or prolonged periods of chest discomfort, acute dyspnoea in the absence of known pulmonary disease, or clinical suspicion of AMI were recruited. Discrimination power was evaluated by calculating the Area under the Receiver-operating characteristics curve; diagnostic performance was assessed at pre-defined

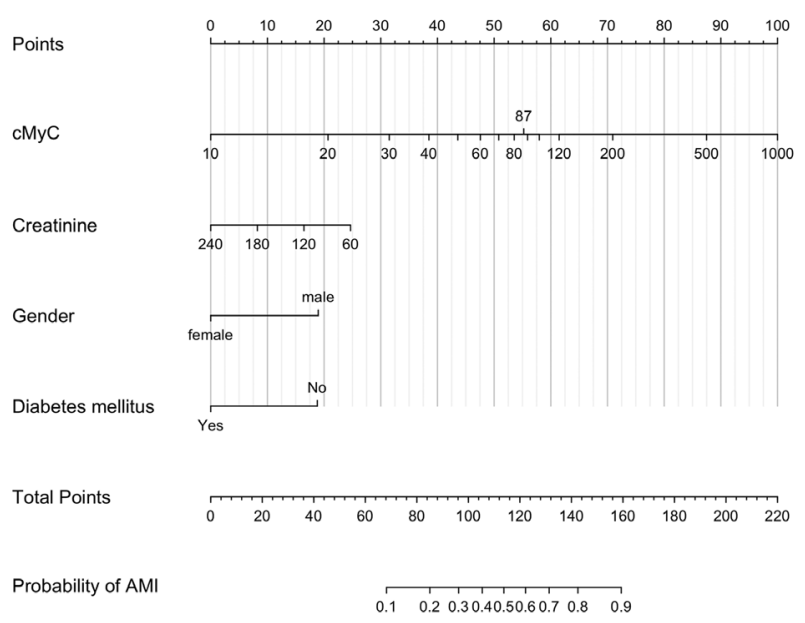

\section{Abstract 148 Figure 1}

thresholds. Diagnostic nomograms were derived \& validated using bootstrap resampling in logistic regression models.

Results 776 patients with median age 68 [58;78] were recruited. AMI was the adjudicated diagnosis in 22\%. Median symptom to sampling time was 70 minutes. cMyC concentration in patients with AMI was significantly higher than with other diagnoses: $98[43 ; 855]$ vs $17[9 ; 42]$ ng/L. Discrimination power for AMI was better with $\mathrm{cMyC}$ than with hscTnT: AUC 0.839 vs $0.813(\mathrm{p}=0.005)$. At a previously published rule-out threshold $(10 \mathrm{ng} / \mathrm{L}), \mathrm{cMyC}$ reaches $100 \%$ sensitivity and NPV in patients after 2 hours of symptoms. In logistic regression analysis, $\mathrm{cMyC}$ is superior to hs-cTnT and was used to derive diagnostic and prognostic nomograms to evaluate risk of AMI and death (figure 1): the nomogram for diagnosis of AMI incorporates easily accessible clinical information plus two biomarker values (cMyC and creatinine) into a probability score for AMI at presentation. When modelling the probability of death during 2-year follow-up, cMyC followed a non-linear curve, with marked variation depending on age and prior myocardial infarction (figure 2).

Conclusion In the prehospital setting, cMyC demonstrates improved diagnostic discrimination of AMI and could significantly improve the early triage of patients with suspected AMI.

Conflict of Interest None

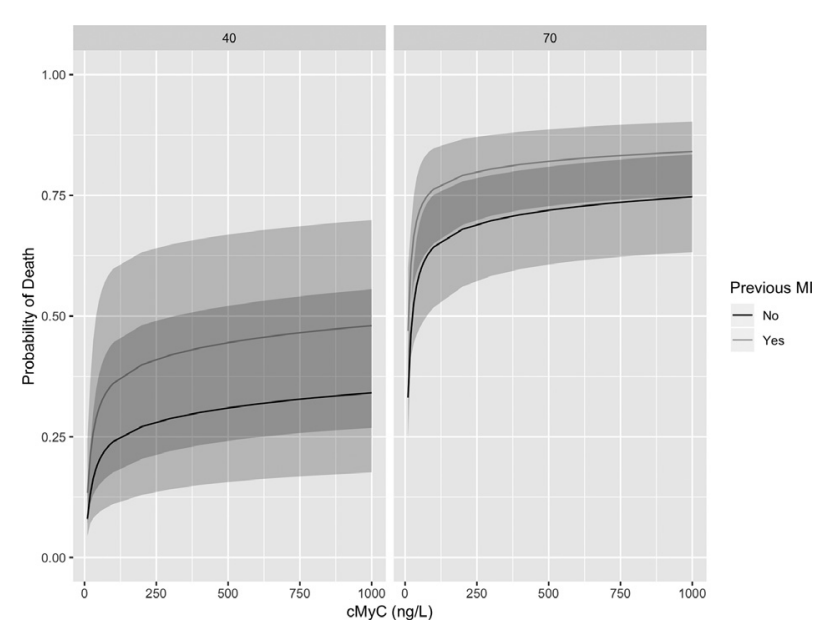

Abstract 148 Figure 2 